\title{
0 PAPEL DO ENSINO A DISTÂNCIA NA EDUCAÇÃO MÉdICA CONTINUADA: UMA ANÁLISE CRÍTICA
}

\author{
luciana Christante*, Monica Parente Ramos, Ricardo Bessa, Daniel Sigulem \\ Trabalho realizado no Laboratório de Ensino a Distância do Departamento \\ de Informática em Saúde da Unifesp, São Paulo, SP.
}

RESUMO - Este artigo é uma revisão de estudos recentes da literatura que avaliaram, sistematicamente, os resultados de programas de educação médica continuada em diversos países, as metodologias de avaliação de demandas específicas nas áreas médicas, e a qualidade e credibilidade dos conteúdos de tais programas. Particularidades do contexto de saúde brasileiro e das tecnologias de internet são descritas, assim como possíveis caminhos para o desenvolvimento da educação médica continuada no Brasil nos próximos anos.

UNITERMOS: Educação. Informática. Medicina. Saúde. Revisão.

\section{INTRODUÇÃO}

Segundo o Accreditation Council for Continuing Medical Education (ACCME) - entidade que certifica provedores de conteúdo para educação médica continuada nos Estados Unidos - o número de médicos registrados em cursos a distância naquele país cresceu I I0\% entre 1998 e $2000^{1-3}$. Apesar do crescimento extraordinário, somente $2 \%$ destes cursos foram oferecidos via internet. Os dados sugerem que, mesmo na principal economia do mundo, o computador e a internet ainda não fazem parte da rotina da grande maioria dos médicos. Mas este fato parece não comprometer 0 crescimento da Educação a Distância $(\mathrm{EaD})$ na área médica. Entre abril de 1999 e janeiro de 200 I surgiram mais de 160 sites de educação médica nos Estados Unidos ${ }^{4}$. Em 1999 os investimentos chegaram a US\$ I, I bilhão, contra apenas US $\$ 650$ mil investidos nos três anos anteriores ${ }^{1-3}$. Mesmo considerando 0 bem conhecido "naufrágio" das chamadas empresas pontocom, ocorrido no final de 2000 , as perspectivas de retorno dos investimentos em $\mathrm{EaD}$ ainda parecem otimistas.

Pela proliferação de escolas e universidades virtuais nos anos recentes, é possível afirmar que os investimentos em $\mathrm{EaD}$, de forma geral, e na área médica em particular, cresce-

*Correspondência: LED-DIS-UNIFESP-EPM

Rua Pedro de Toledo, $781,2^{\circ}$ andar. 04039-032- São Paulo - SP luciana@dis.epm.br ram também no Brasil, embora não haja dados precisos a respeito. As pressões que impulsionam o desenvolvimento dessa área advêm, por um lado, do recente e acelerado desenvolvimento tecnológico e, por outro, da velocidade cada vez maior com que o conhecimento científico se torna obsoleto. Procurar, entender e utilizar informações técnicas e científicas, agora disponíveis numa escala sem precedentes, é de fundamental importância para todas as áreas profissionais, em especial para a Medicina.

$\mathrm{A} E \mathrm{ED}$ é tão antiga quantos os Correios, mas foi com a explosão da internet que ela se tornou mais atraente como forma de promover educação profissional continuada, uma necessidade emergente a partir da década de 90. No mundo todo, a Educação Médica a Distân$\mathrm{cia}(\mathrm{EMaD})$ vem ganhando mais espaço à medida que aumentam os programas de garantia de qualidade, recertificação e acreditação. No Brasil o tema é relativamente recente, mas em diversos países da Europa, nos Estados Unidos eno Canadá, programas de $\mathrm{EMaD}$ (via internet ou não) existem há pelo menos 10 anos ${ }^{5}$.

A situação do setor de saúde brasileiro é certamente bem mais complexa que a dos países desenvolvidos. Diante das dimensões territoriais de nosso país, da concentração de riqueza e conhecimento nas regióes sudeste e sul, das iniciativas para melhorar a qualidade do ensino médico e da relação médico-paciente, a EmaD parece ser um mecanismo que, se bem planejado e bem-sucedido, poderia ajudar a corrigir ou amenizar algumas destas distorções.
Uma pesquisa da Fundação Oswaldo $\mathrm{Cruz}^{6}$ mostrou que a classe médica tem disposição para atualizar e aumentar seus conhecimentos, no entanto, o gradiente regional característico do Brasil é um fator limitante. Os dados mostraram que $72 \%$ dos médicos com curso de especialização estão concentrados nas capitais brasileiras, contra $27,8 \%$ que residem no interior. $A$ $\mathrm{EMaD}$ pode atingir profissionais que, por restrições econômicas ou de tempo, não podem participar de eventos científicos, congressos nacionais ou internacionais e, além disso, pode contribuir para que a excelência de diversos centros de ensino e pesquisa possa alcançar regiões distantes do país - provavelmente as que dela mais necessitam.

Em que pesem as dificuldades de acessoà internet por grande parte da população brasileira, a oferta de cursos a distância para médicos é crescente. Menos enfatizadas são a demanda real e a eficácia dessa modalidade de ensino. Neste artigo procuramos nos ocupar destas últimas duas questões por considerá-las pouco discutidas no nosso meio. A literatura científica internacional sobre o tema já possui uma boa coleção de exemplos e reflexões sobre os objetivos e resultados de programas de $\mathrm{EMaD}$ em outros países. Acreditamos que vale a pena conhecê-los - guardadas, obviamente, as devidas particularidades de cada contexto. Não se trata de uma revisão sistemática, mas de uma primeira aproximação ao tema; esperamos que outros possam criticá-la eaperfeiçoá-la. 
Neste artigo os termos "educação médica continuada" (EMC) e "educação médica a distância" (EMaD) serão usados como sinônimos. Embora o primeiro não necessariamente envolva o uso de tecnologias como a internet, a maioria das iniciativas da atualidade as usa de forma complementar ou exclusiva.

\section{Objetivos e avaliação}

Da mesma forma que os médicos intervêm na doença para mudar o estado de saúde dos pacientes, o objetivo da EMCé intervir nos aspectos da prática médica que possam ser melhorados. Para Fox \& Bennet $^{5}$, a EMaD é uma tentativa sistemática de facilitar mudanças na prática clínica, devendo assim deixar seu enfoque meramente instrucional para estar mais voltada à facilitação do aprendizado. Para isso é preciso, em primeiro lugar, conhecer as necessidades e motivações específicas facilitadoras do aprendizado. Essa mudança de paradigma pode também, segundo os autores, dar a oportunidade para refletir criticamente e experimentar as inovações da Medicina antes de adotá-las na prática clínica.

Nesta mesma linha, Cantillon \& Jones ${ }^{7}$ declaram que o objetivo principal da EMC deve ser o aperfeiçoamento da prática clínica. Em outras palavras, deve ser usada como uma estratégia de intervenção educacional. Eles ressaltam uma mudança na base de conhecimento que reflete a incorporação de teorias de aprendizado em adultos, que por sua vez reconhecem que é o aprendizado - e não o ensino - que é capaz de transformar a prática profissional. Para tanto, as estratégias de intervenção educacional devem estar fortemente direcionadas ao trabalho cotidiano dos profissionais. Em excelente revisão sistemática sobre o tema, estes autores relatam um grande número de intervenções educacionais que melhoraram não só o desempenho dos médicos, mas também os desfechos clínicos. Um exemplo é um estudo sueco ${ }^{8}$ no qual fica evidente $\mathrm{o}$ aspecto "continuado" da EMaD. Através de múltiplas estratégias de ensino e comunicação (entre elas a internet) para melhorar o atendimento médico de pacientes com depressão em uma pequena cidade sueca, os resultados foram tão positivos que promoveram redução na taxa de suicídio. No entanto, nova avaliação feita três anos depois mostrou deterioração do desempenho médi-

\section{Figura I - Hierarquia de Kirkpatrick ${ }^{25}$ para níveis de avaliação. A complexidade da avaliação é maior no topo da pirâmide. $O$ impacto na sociedade não pode ser atingindo sem que as etapas anteriores sido bem-sucedidas}

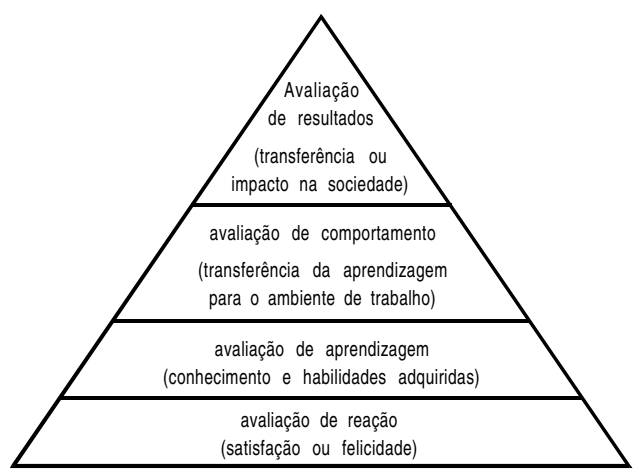

co e retorno da taxa de suicídio aos níveis anteriores à intervenção - 0 autor salienta a importância do reforço no aprendizado. Dos 69 estudos incluídos na revisão sistemática de Cantillon \& Jones ${ }^{7}$, apenas quatro realizaram reavaliações depois de, no máximo, três meses. Com esses resultados, os autores mostram que o aspecto continuado da intervenção educacional não se deve aplicar apenas à continuidade de oferta de programas educacionais, mas também a um planejamento em longo prazo de reforço e avaliação dos efeitos de cada programa.

Diversos outros estudos sobre a eficiência $\mathrm{da}$ EMaD foram publicados nos últimos anos 9 17. Hutchinson ${ }^{9}$ propõe que, assim como na pesquisa clínica, o embasamento em evidências científicas é essencial para a avaliação educacional e sugere $o$ termo educação médica baseada em evidências para a construção de um novo paradigma na EMC. A autora aponta para a complexidade dos fatores que podem influenciar a eficiência de tais intervenções (por exemplo, experiência prévia e motivação) e para uma hierarquia de níveis de avaliação, na qual a complexidade da mudança de comportamento aumenta conforme a avaliação ascende a hierarquia (Figura I).

Campbell \& Johnson ${ }^{10}$ analisaram a combinação de diferentes tipos de mídia, sobretudo o poder do computador e a incorporação de tecnologias de comunicação em intervenções a distância. Seus resultados mostraram que inovações como a internet e CD-ROM podem fornecer condições de acesso e interatividade relevantes e de baixo custo. No entanto, alertam que a falta de objetivos bem definidos pode fazer da $\mathrm{EMaD}$ apenas uma estratégia de massa que pode resultar em baixo aproveitamento de grandes coleções de versões eletrônicas de material didático-em outras palavras, uma interessante mudança nos "meios", mas sem grandes alterações nos "métodos".

Apesar da existência de bons estudos e reflexões críticas sobre $o$ assunto, a maior parte das publicações nessa área carece de metodologias adequadas ou apresenta lacunas conceituais. Segundo Cantillon \& Jones ${ }^{7}$, o principal problema desses estudos é a ausência de avaliações objetivas. As conseqüências são resultados genéricos e exageradamente otimistas. Para eles, os responsáveis por programas educacionais costumam gastar seus limitados recursos mais para desenvolver e executar inovações educacionais do que para avaliá-las. Além disso, estudos de avaliação educacional raramente são publicados porque, em geral, são rejeitados por não serem suficientemente rigorosos. As pesquisas controladas são particularmente difíceis e há problemas para encontrar grupos-controle apropriados. Contudo, apesar das dificuldades, a avaliação continua sendo uma parte importante do ciclo educacional e deve estar incluída em qualquer estratégia de EMC. Os mesmos princípios aplicados à Medicina baseada em evidências podem ser aproveitados para o desenvolvimento de programas educacionais naárea médica.

\section{Avaliação da demanda}

Se por um lado o acompanhamento rigoroso dos resultados de um programa de $\mathrm{EMaD}$ 
é necessário para sua eficiência, por outro, há que se tomar cuidado com as avaliações prévias sobre a demanda, requisito indispensável para conduzir e dirigir o processo educacional. Por avaliação de demanda entenda-se a forma como se elege os temas mais relevantes que serão foco de uma determinada intervenção. $\mathrm{Na}$ área de saúde, o leque de assuntos e enfoques é inumerável e muitos esforços e recursos podem ser poupados ou otimizados se a proposta de intervenção estiver afinada às necessidades do público-alvo.

Cabe ressaltar a problemática relacionada à percepção dos médicos sobre suas próprias necessidades educacionais. Tracey et al. " observaram uma fraca correlação entre a autoavaliação dos conhecimentos de médicos da Nova Zelândia e subseqüentes performances em testes objetivos dos mesmos conhecimentos. Segundo os autores, os programas educacionais baseados na auto-aprendizagem e na contínua melhoria da qualidade são teoricamente bons e um método valioso da aprendizagem, mas salientam que, se a avaliação inicial das próprias necessidades de aprendizagem for deficiente, todo o sistema será fundamentalmente afetado.

Resultados semelhantes foram obtidos por Sibley et al. ${ }^{12}$ em um estudo controlado feito no Canadá. Dada a oportunidade de os médicos escolherem conteúdos educacionais, a tendência foi pela escolha de assuntos que eles já dominavam - e neste caso, o efeito da intervenção sobre o desempenho clínico não foi diferente em relação ao grupo-controle. Já quando os tópicos abordados não estavam entre as preferências dos clínicos, o desempenho melhorou significativamente após a intervenção, em relação ao grupo-controle. Violato et al. ${ }^{13}$ alertam que a auto-avaliação dos médicos pode ser usada para avaliação da demanda, mas não exclusivamente e sim em combinação com evidências provenientes de outras fontes, como sistemas de avaliação por pares, médicosconsultorese, inclusive, pacientes.

Para Tracey et al. " duas razões poderiam explicar a dificuldade dos médicos para avaliar seu conhecimento. A primeira é a natureza solitária de seu trabalho, que lhe dá poucas oportunidades de discussões regulares com seus pares; a segunda éa rápida velocidade da produção científica, que torna muito difícil para os médicos saber quando seus conhecimentos se tornam desatualizados. Segundo estudo metodológico feito por Jansen et al. ${ }^{17}$, quanto mais específica é a área de atuação do clínico, mais deficiente é sua auto-avaliação.

\section{Qualidade e CRedibiLidade}

Outro fator crítico para o estabelecimento de um programa de EMC de alta qualidade é a confiabilidade técnica dos conteúdos. As dúvidas que pairam em relação à privacidade dos usuários na internet parecem ser o principal fator responsável pela falta de credibilidade. Os usuários se apresentam mais temerosos em relação a sites com finalidades comerciais, o que podeser explicado pela falta de normaséticase leis aplicáveis ao setor e pela incerteza sobre quem regula a internet. Este assunto está mais bem documentado em relação asites de saúde voltados aos leigos e, por esta razão alguns levantamentos são citados aqui, se supondo que tendências semelhantes podem ser encontradas em relação à educação profissional. Uma pesquisa da California Healthcare Foundation ${ }^{18}$ revelou que, embora a maioria dos sites comerciais de saúde declare políticas de responsabilidade e privacidade, estas raramente são seguidas. Berland et al. ${ }^{19}$ apontam uma série de problemas de qualidade, acessibilidade e legibilidade em sua pesquisa que cobriu 25 sites de língua inglesa e espanhola nos Estados Unidos. Questões como autoria, validação e sistemas de avaliação por pares são essenciais para uma discussão apropriada sobre qualidade e fidedignidade de conteúdos educacionais.

Uma possibilidade para evitar distorções na qualidade dos conteúdos oferecidos tantoa pacientes quanto a profissionais de saúde é o estabelecimento de protocolos que norteiem esse tipo de publicação. Um exemplo são os Guidelines for medical and health information on the internet ${ }^{20}$ elaborados pela American Medical Association (AMA). Trata-se de um documento que estabelece regras para criação e submissão (avaliação por pares) de conteúdos para EMaD e para leigos, bem como princípios éticos para concessão de apoios e patrocínios do setor privado. Dessa forma, a AMA funciona como uma entidade acreditadora da qualidade dos conteúdos de saúde na internet naquele país. No Reino Unido iniciativas semelhantes são promovidas pelo British Medical Journal ${ }^{21}$ em parceria com a British Medical Association.
Contudo, a mais bem conhecida iniciativa de estabelecer padrões de conduta para sites de saúde na internet é a da Health On The Net Foundation (HON), uma organização não governamental internacional com sede na Suíça e que estabeleceu o HONCode ${ }^{22}$, o código de conduta mais citado na web quando 0 tema é saúde. Seguindo os princípios do HONCode, o Conselho Regional de Medicina do Estado de São Paulo (Cremesp) criou a resolução 097/200I que estabelece manual de ética ${ }^{23}$ para sites de saúde e medicina na internet. Mas tanto o HONCode quanto o manual do Cremesp não se referem a conteúdos de $E a D$ na área de saúde nem tampouco a mecanismos de avaliação e certificação periódica de todo tipo de conteúdo, seja para leigos, seja para profissionais. Assim, não há como assegurar a qualidade do produto final e, logo, a confiança e o respeito dos usuários. As entidades reguladoras e representativas da classe médica, como a Associação Médica Brasileira, o Conselho Federal de Medicina poderiam contribuir com a criação de protocolos para $\mathrm{a} \mathrm{EMaD}$, à semelhança do que fazem com protocolos próprios de cada especialidade médica.

\section{Considerações finais}

O Brasil é o país da América Latina que mais tem feito esforços para não ficar atrás na corrida tecnológica. Em outubro de 200I, havia quase 6 milhões de usuários domiciliares conectados à internet ${ }^{24}$. Nosso país possui inúmeros centros de excelência em ensino e pesquisa na área médica, ainda que estes estejam demasiadamente concentrados nas regiões sul e sudeste. Logo, a EMaD pode vir a ser um meio para permitir que essa excelência possa ser mais bem distribuída pelo país, facilitando o acesso ao conhecimento e ao aperfeiçoamento profissional daqueles que residem em áreas distantes e cujas populações sofrem ainda mais com a má qualidade da assistência à saúde.

No Brasil, a EMaD vem se desenvolvendo em diversas instituições de ensino superior. A Universidade Federal de São Paulo/Unifesp (www.unifesp.br), por ser uma universidade temática da saúde e reconhecido centro excelência em ciências médicas, tem na Unifesp Virtual a missão de 
levar o conhecimento médico através da educação a qualquer lugar do país. A Unifesp Virtual, hoje mantida pelo Laboratório da Educação a Distância, é o resultado do amadurecimento do grupo de educação do Departamento de Informática em Saúde desde o fim da década de 80 . Hoje, com apenas dois anos de existência oficial, produz e mantém, em parceria com departamentos acadêmicos e a comunidade, diversos cursos on-line e materiais de apoio ao aprendizado para profissionais e público geral. Os dados e resultados destas experiências vêm sendo foco de dissertações de mestrado, teses de doutorado e outros tipos publicação, no esforço de avaliar a qualidade e o impacto da EMaD em nosso meio.

Neste artigo, procuramos abordar criticamente o papel do ensino a distância na educação médica continuada com o intuito de incentivar 0 amadurecimento e a evolução dessa área no Brasil. Em suma: objetivos claros e resultados mensuráveis, demandas criteriosamente avaliadas e credibilidade dos provedores de conteúdo são questões-chave para que a EMaD possa comprovadamente trazer aperfeiçoamento à prática clínica e benefícios concretos à assistência à saúde da população brasileira.

\section{SUMMARY}

The ROLE OF DISTANCE EDUCATION IN CONTINUING MEDICAL EDUCATION: A CRITICAL REVIEW

This paper reviews findings of recent literature which have systematically evaluated outcomes of continuing medical education programs worldwide, methods for measuring especific demands in medical areas, and quality and reliability of contents related to such programs. Particular features of the Brazilian health context and internet technologies are described, as well as possible directions towards life-long medical education development in Brazil for the next few years. [Rev Assoc Med Bras 2003; 49(3): 326-9]

Key words: Education. Informatics. Medicine. Health. Review.

\section{REFERÊNCIAS}

I. ACCME. Anual Report Data 1998. Available from: URL: http://www.accme.org/incoming/ 1998_annual_data_analusis_for_website.pdf.

2. ACCME. Annual Report Data 1999. Available from:URL: http://www.accme.org/incoming/ 1999_annual_data_analysis_for_website.pdf.

3. ACCME. Annual Report Data 2000. Available from: URL: http://www.accme.org/incoming/ 2000_annual_data_analysis_for_website.pdf.

4. Sklar BM. The current status of online continuing medical education. A master's thesis in medical information science. San Francisco: University of California; 2001. Available from: URL: http://netcantina.com/ masterthesis.

5. Fox RD, Bennet NL. Learning and change: implications for continuing medical education. BMJ 1998; 316:466-8. Available from: URL http://bmj.com.cgi/content/full/316/7/29/466.

6. Machado, MH. Perfil dos médicos do Brasil 1996. Escola Nacional de Saúde Pública, Fundação Oswaldo Cruz. Available from: URL: http://www.ensp.fiocruz.br/perfil.

7. Cantillon $P$, Jones R. Does continuing medical education in general practice can make a difference? BMJ 1999;3 18:1276-9. Available from: URL: http://bmj.com/cgi/content/full/ 318/7/93/1276.

8. RutzW, Von Knorring L, Walinder J. Long-term effects of an educational program for general practitioners given by the Swedish committe for the prevention and treatment of depression. Acta Psychiatr Scand 1991;85:83-8.

9. Hutchinson L. Evaluating and researching the effectiveness of educational interventions. BMJ 1999; |8: | 275-9. Available from: URL: http:// bmj.com/cgi/content/full/3 /8/7/93// 267.

10. Campbell JK, Johnson C. Trend spotting: fashions in medical education. BMJ 1999; 18: 1272-5. Available from: URL: http:// bmj.com/cgi/content/full/3 I8/7/93// 272.

II. Tracey J, Arrol B, Barhan P, Richmon D. The validity of general practitioner's self assessment of knowledge: cross sectional study. BMJ 1997;3 15: 1426-8. Available from: URL: http:/ /bmj.com/cgi/content/full/3 I5/7/20//426.

I2. Sibley JC, Sackett DL, Neufeld V, Gerrard B, Rudnick KV, FraserWA. A randomized trial of continuing medical education. $N$ Engl J Med 1982; 315:5।I-5.

13. Violato C, Marini A, Toews J, Lockyer J, Fidler $H$. Feasebility and psychometric properties of using peers, consulting physicians, co-wokers, and patients to assess physicians. Acad Med 1997; 72:S82-4.

14. Towle A. Continuing medical education: changes in health care and continuing medical education. BMJ 1998;316-30I-4. Available from: URL: http://bmj.com/cgi/content/full/ $3167 / 7127 / 301$.
15. Mack M. Points for reading as part of continuing medical education. BMJ 2000;320: I408- 10. Available from: URL: http://bmj.com/cgi/ content/full/320/7246/I408/a.

16. Holm HA. Should doctors get CME points for reading? BMJ 2000;320:394-5. Available from: URL: http://bmj.com/cgi/content/full/320/ 7232/394.

17. Jansen JJM, Tan LHC, van der Vieuten CPM, van Luijk SJ, Rethans JJ, Grol RPTM. Assessment of competence in technical clinical skills og general practitioners. Med Educ 1995; 29:247-53.

18. Goldman J, Hudson Z,Smith RM. Ethics survey of consumer attitudes about health web sites. Californica Heathcare Foundation 2000. Available from: URL: http://ehealth.chcf.org.

19. Berland GK, Elliot MN, Morales LS, Algazy Jl, Kravitz RL, Broder MS et al. Health quality on the internet: accessibility, quality, and readability in English and Spanish. JAMA 200 I 285:26|2-2|

20. Winker MA, Flanagin RN, Chi-Lum B, White J, Andrews $\mathrm{K}$, Kennet RL et al. Guidelines for medical and health information sites on the internet. American Medical Association 2001. Available from: URL: http://www.amaassn.org/ama/pub/category/ 1 905.html.

21. Education Group for Guidelines on Evaluation. Guidelines for evaluating papers on educational interventions. BMJ 1999;318: | 265-| 267. Available from: URL: http://bmj.com/cgi/content/full/3 I8/7 I 93/ 1265.

22. Health On the Net Foundation. Código de conduta para sites web de medicina e saúde. Available from: URL: http://www.hon.ch/ HONcode/Portuguese/.

23. CREMESP. Manual de ética para sites de medicina e saúde na internet. 200I. Disponível em: URL: http://www.cremesp.org.br/ legislacao/leg_internet/etica_internet.htm.

24. Jupiter Media Metrix. Relatório de audiência de mídia digital domiciliar. Outubro de 200I.Avaliable from: URL: http:// br.mediametrix.com/xp/br/press/releases/ pr_III $201 . x m l$.

25. KirkpatrickDI. Evaluation of training. In: Craig R, Bittel I, editors. Training and development handbook. New York: McGraw-Hill , 1967 (apud Hutchinson L. Evaluating and researching the effectiveness of educational interventions. BMJ 1999; I8: 1 275-9. Available from: URL: http://bmj.com/cgi/content/full/ 318/7/93//267)

Artigo recebido: 22/05/02 Aceito para publicação: 18/03/03 